\title{
Favorable revascularization therapy in patients with ASPECTS $\leq 5$ on DWI in anterior circulation stroke
}

\author{
Isabelle Mourand, ${ }^{1}$ Eitan Abergel, ${ }^{2}$ Daniel Mantilla, ${ }^{3}$ Xavier Ayrignac, ${ }_{1}^{1}$ Tzvika Sacagiu, ${ }^{3}$ \\ Omer Faruk Eker, ${ }^{3}$ Gregory Gascou, ${ }^{3}$ Cyril Dargazanli, ${ }^{3}$ Carlos Riquelme, ${ }^{3}$ \\ Marinette Moynier, ${ }^{3}$ Alain Bonafé, ${ }^{3}$ Caroline Arquizan, ${ }^{1}$ Vincent Costalat ${ }^{3}$
}

${ }^{1}$ Department of Neurology, CHRU Gui de Chauliac, Montpellier, France

${ }^{2}$ Department of Neuroradiology, Rambam Health Care Campus, Haifa, Israel

${ }^{3}$ Department of Neuroradiology, CHRU Gui de Chauliac, Montpellier, France

\section{Correspondence to}

Dr Isabelle Mourand, Neurology Department, CHRU, Gui de

Chauliac Hospital, 34295

Montpellier, France; i-mourand@ chu-montpellier.fr

IM and EA contributed equally.

Received 3 August 2017 Revised 4 October 2017 Accepted 5 October 2017

Published Online First

27 October 2017

\section{CrossMark}

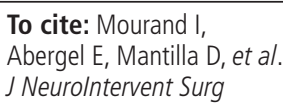

ABSTRACT

Background A low baseline Alberta Stroke Programme Early CT Score (ASPECTS) is strongly associated with low rates of favorable outcome in patients with acute stroke. Objective To evaluate the efficacy and safety of revascularization therapy in patient with ASPECTS $\leq 5$ in anterior circulation infarct.

Methods We retrospectively analyzed 108 consecutive patients presenting low ASPECTS on diffusion-weighted imaging. Sixty patients were treated by mechanical thrombectomy, including 34 patients who received simultaneously intravenous thrombolysis. A control group of 48 patients not eligible for reperfusion therapy gave us a perspective on the natural history. Clinical outcome was evaluated at 90 days using the modified Rankin Scale (mRS) score. Hemicraniectomy after malignant infarction, mortality, and symptomatic intracranial haemorrhage $(\mathrm{s} \mid \mathrm{CH})$ were also reported.

Results Thrombolysis in Cerebral Infarction 2b-3 was assessed in $75 \%$ of treated patients. Reperfusion therapy led to significantly reduced disability (mRS score $0-2$ ) at 90 days compared with the control group (30\% vs $2.1 \%$, $\mathrm{p}<0.001)$, hemicraniectomy (3.3\% vs $22.9 \%, \mathrm{p}=0.002)$, and death at 90 days ( $25 \%$ vs $47.9 \%, p=0.01)$. The sICH level was similar in treated patients and in the control group ( $p=0.78$ ). Patients aged $\leq 70$ years in the thrombectomy group had a significantly better clinical outcome than older patients $(37.5 \%$ vs $10 \%, p=0.02)$, regardless of baseline characteristics or recanalization rate.

Conclusions In patients with acute stroke in the anterior circulation and ASPECTS $\leq 5$ revascularization therapy contributes to a favorable clinical outcome at 90 days, especially in patients younger than 70 years.

\section{INTRODUCTION}

The infarct volume at baseline is a strong and independent predictor of clinical outcome at day $90^{1}$ and a critical determinant in patients treated with reperfusion therapies. ${ }^{2}$ Similarly, SAMURAI recombinant tissue plasminogen activator registry demonstrated that Alberta Stroke Programme Early CT Score (ASPECTS) $\leq 4$ on diffusion-weighted imaging (DWI) was related to death at 3 months and $\leq 5$ to symptomatic intracranial haemorrhage (sICH) within 36 hours. $^{3}$

In 2015, several major randomized trials ${ }^{4-8}$ proved that mechanical thrombectomy combined with IV thrombolysis (IVT) was better than IVT alone in the treatment of stroke with large vessel occlusion. The primary end point of these studies focused on disability reduction at 3 months, with modified Rankin Scale (mRS) score $\leq 2$ ranging from $33 \%$ to $72 \%{ }^{7}$ in the thrombectomy arm with a consistent increase in the absolute value. To achieve these results, patient selection based on imaging was mandatory, excluding patients with a large infarction core. In the meta-analysis of the HERMES collaborators, ${ }^{9}$ low baseline ASPECTS is strongly associated with low rates of favorable outcome.

Unfortunately, real-world patients are not always the ideal 'trial' candidates for thrombectomy. Therefore, neurologists and interventional neuroradiologists are confronted daily with the decision to treat or not patients with acute stroke and a large necrotic core admitted in the recanalization time window with an accessible thrombus for thrombectomy. For this situation, no therapeutic criteria guidelines are available.

The aim of this study was to analyse the efficacy and safety of revascularization therapy using mechanical thrombectomy (MT) or bridging therapy in patients presenting with a large necrotic core stroke in the anterior circulation based on ASPECTS $\leq 5$ on DWI. Our hypothesis was that revascularization might be beneficial in patients with DWI ASPECTS $\leq 5$, resulting in reduced disablity at 3 months, and lower mortality and malignant infarct.

\section{METHODS}

\section{Study design}

Our study retrospectively analyzed patients admitted to our neurovascular unit between January 2009 and December 2014.

Data of patients in the treatment group were taken from our thrombectomy database. Our selection criteria were (1) acute ischemia lesions within the anterior circulation on DWI; (2) ASPECTS $\leq 5$ on apparent diffusion coefficient (ADC) cartography; (3) presence of a proximal intracranial artery occlusion in the stroke territory; (4) presence of a clinical-diffusion mismatch, defined by the presence of a neurological deficit unexplained by the infarction lesions seen on ADC mapping; (5) National Institute of Health Stroke Scale (NIHSS) score $\geq 8$; (6) initiation of endovascular treatment within 8 hours of onset of the first symptom; (7) pre-stroke mRS score $\leq 1$. Consent for treatment was obtained from the patients or their family members before the endovascular procedure. Patients in the treatment 
group received intravenous alteplase within 4.5 hours after the onset of stroke symptoms if they met the guideline criteria. ${ }^{10}$

The control group comprised patients with a similar ASPECTS range during the same period of time but who did not met selection criteria for revascularization therapy.

Details of basic demographics and cardiovascular risk factors were available for all patients. Admission NIHSS score assessment was performed by a stroke neurologist. Information on the delay from onset of symptoms to presentation at the emergency room and delay from onset of symptoms to MRI was collected.

\section{Imaging protocol}

All patients underwent acute multimodal $3 \mathrm{~T}$ (Maqueton, Skyra, Siemens, Erlangen, Germany) or 1.5 T MRI (Avanto, Siemens, Germany). A standardized imaging protocol was performed in the ischemic acute phase, including DWI (TR/TE $=3600 / 83 \mathrm{~ms}, \mathrm{~b}$ values, 0 and $1000 \mathrm{~s} / \mathrm{mm}^{2}$ isotropic, voxel size $\left.1.8 \times 1.8 \times 5 \mathrm{~mm}\right)$, reconstruction of ADC mapping, fluid-attenuated inversion recovery, T2* gradient echo sequences, intracranial and cervical gadolinium-enhanced MR angiography. Perfusion-weighted imaging was not acquired systematically. To evaluate the extension of the ischemic lesion on ADC mapping, ASPECTS was used. ASPECTS was calculated on the ADC map by an independent neuroradiologist blinded to the angiographic data. We used the $1 / 3$ rule-that is, a region is counted if the infarct on MRI comprises more than one-third of the ASPECTS region.

\section{Revascularization protocol}

IVT $(0.9 \mathrm{mg} / \mathrm{kg})$ was administered to patients within 4.5 hours after onset of the first symptoms. Conventional clinical and laboratory inclusion and exclusion criteria for IVT thrombolysis were applied. ${ }^{10}$ In cases of bridging therapy, patients were transferred to the angiographic suite for thrombectomy as soon as possible. Among patients with contraindication to IVT, thrombectomy alone was performed. MT was performed via a femoral artery approach under general anesthesia with available thrombectomy devices. The choice of thrombectomy device was left to the operator. An $8 \mathrm{~F}$ or 9F Merci balloon-guide catheter (Concentric Medical) was inserted through a sheath. A 0.21 inch internal diameter microcatheter (Prowler Select Plus; Cordis or Vasco 21; Balt) was navigated distal to the occlusion over a 0.014 inch steerable guidewire, which was then exchanged with the thrombectomy device. During the retrieval, the balloonguide catheter was inflated to interrupt anterograde flow. Manual aspiration with a $50 \mathrm{~mL}$ syringe was performed through the hemostatic valve during the retrieval, to reverse the flow and to aspirate clot debris possibly lost in the guide catheter lumen. Attempts to retrieve the thrombus were limited to five passes by occluded vessel. Follow-up CT or MRI was performed 24 hours after MT in order to assess the extent of the infarction and/or hemorrhagic complications. If no hemorrhage was present, antiplatelet drugs were administered.

\section{Outcome measures}

In the treatment group, successful reperfusion was defined by a Thrombolysis in Cerebral Infarction (TICI) score of 2b-3 assessed at the end of the procedure. Delay from symptoms onset to desobstruction as well as delay from MRI to desobstruction was calculated for the thrombectomy group.

The mRS score at 90 days was assessed for all patients by a trained neurologist. Favorable outcome was defined as a mRS score $\leq 2$. A mRS score of 6 was attributed to a patient lost to follow-up. sICH was defined as a documented hemorrhage on
Table 1 Baseline characteristics of the thrombectomy group $(n=60)$ and the control group $(n=48)$

\begin{tabular}{|c|c|c|}
\hline Baseline characteristics & $\begin{array}{l}\text { Thrombectomy } \\
(n=60)\end{array}$ & Control $(n=48$ \\
\hline Median age (IQR) & $66(22-86)$ & $67(41-87)$ \\
\hline Male, n (\%) & $40(66.7 \%)$ & $30(62.5 \%)$ \\
\hline Female, n (\%) & $20(33.3 \%)$ & $18(37.5 \%)$ \\
\hline Hypertension, n (\%) & $28(46.7 \%)$ & $23(47.9 \%)$ \\
\hline Dyslipidemia, n (\%) & $19(31.7 \%)$ & $15(31.3 \%)$ \\
\hline Diabetes, n (\%) & $11(18.3 \%)$ & $9(18.8 \%)$ \\
\hline Previous CVA, $n(\%)$ & $22(36.7 \%)$ & $16(33.3 \%)$ \\
\hline Initial median NIHSS (IQR) & $20(9-28)$ & $22(5-40)$ \\
\hline Median ASPECTS (IQR) & $5(2-5)$ & $3(0-5)$ \\
\hline \multicolumn{3}{|l|}{ Occluded artery, n (\%) } \\
\hline MCA M1 & $34(56.7 \%)$ & $27(56.3 \%)$ \\
\hline MCA distal & $5(8.3 \%)$ & $3(6.3 \%)$ \\
\hline Tandem & $13(21.7 \%)$ & $9(18.8 \%)$ \\
\hline $\mathrm{T}$ carotid & $8(13.3 \%)$ & $9(18.8 \%)$ \\
\hline \multicolumn{3}{|l|}{ Median delay, $\min (\mathrm{IQR})$} \\
\hline Symptoms, onset to admission & $140(10-380)$ & $171(37-642)$ \\
\hline Symptoms, onset to MRI & $164(20-392)$ & $172(58-667)$ \\
\hline Symptoms, onset to recanalization & $327(153-514)$ & NA \\
\hline MRI to recanalization & $132(55-382)$ & NA \\
\hline
\end{tabular}

ASPECTS, Alberta Stroke Programme Early CT Score; CVA, cardiovascular accident; IQR, interquartile range; MCA, middle cerebral artery; NIHSS National Institute of Health Stroke Scale.

CT or MRI, with a decline of $\geq 4$ points in the NIHSS score. Safety outcomes also included malignant stroke, defined as rapid neurological deterioration due to the effects of space-occupying cerebral edema following middle cerebral artery (MCA) territory stroke, decompressive craniectomy in malignant MCA infarction, and mortality at 90 days.

\section{Statistical analysis}

Three subgroup analyses were performed. In the first, we compared patients in the treatment group with patients in the control group. A second subgroup analysis was based on age, with a 70-year-old cut-off point. In the third analysis, we subdivided our study population into two ASPECTS groups: one with a very low ASPECTS (0-3) and the second with a low ASPECTS (4-5). Patient demographics, occlusion site, and stratification based on time and imaging are descriptively presented and compared. The significance of simple bivariate associations was assessed using Student's t-test.

\section{RESULTS}

\section{Baseline clinical and imaging characteristics}

Baseline clinical and imaging characteristics are shown in table 1.

Sixty patients were included in the treatment group and 48 in the control group. Patients in the control group were excluded from thrombectomy mainly because of the lack of a clinical-diffusion mismatch (40 patients; $83 \%$ ). Other reasons for exclusion were: NIHSS $<8$ for one patient, admission in our stroke center more than 8 hours after the onset of symptoms for three patients, pre-stroke mRS score $>1$ for four patients. The main contraindication for IVT was an infarct greater than one-third of the MCA territory (25 patients, 52\%). Other contraindications were: 
admission to our stroke unit $\geq 4.5$ hours after onset of symptoms (eight patients), wake-up stroke (six patients), warfarin therapy with international normalized ratio (INR) $>1.7$ (four patients), hemorrhage transformation in the infarct area on presentation (three patients), cavernoma (one patient), and surgery within 30 days (one patient).

Median NIHSS score at admission was 20 in the treatment group versus 22 in the control group. The median delay from onset of symptoms to presentation to the emergency room was higher, though not significantly, in the control group than in the treatment group ( $171 \mathrm{~min}$ vs $140 \mathrm{~min} ; \mathrm{p}=0.56)$. Similarly, the median delay from the onset of symptoms to MRI was not significantly different in the control group ( $172 \mathrm{~min})$ compared with the treatment group (164 min, $\mathrm{p}=0.15)$. The median ASPECTS in the treatment group and in the control group were significantly different (5 vs 3 respectively; $\mathrm{p}<0.001)$. Fifty-four patients (90\%) in the treated group and 21 patients (44\%) in the control group had a baseline ASPECTS of 5 or 4 . In the treatment group, $22 \%$ of the patients had a tandem lesion versus $19 \%$ in the control group. Infarct etiology of the 13 tandem occlusions in the thrombectomy group, according to the TOAST (Trial of Org 10172 in Acute Stroke Treatment) classification, was internal carotid dissections in four patients (31\%), atherothrombotic in seven (54\%), cardioembolic in one and undetermined in one. Patients with an atherothrombotic tandem occlusion were older than patients with internal carotid dissection (67 years vs 51.5 years; median) and presentation NIHSS score was higher (21 vs 14.5) with equal ASPECT score (5 vs 5).

Among the treatment group, 34 patients (56.7\%) received bridging therapy and 26 patients $(43.3 \%)$ thrombectomy alone. Fifty-three patients (88\%) were treated with the Solitaire FR device (Medtronic, Irvine, USA). The other treatment modalities included the Trevo XP (Stryker Neurovascular, Freemont, USA) for three patients, the Catch (Balt Extrusion, Montmorency, France) for three patients and the Merci (Concentric Medical, Mountain View, USA) for one patient. The median number of passes with the thrombectomy device was 2 (range 1-5). TICI $2 \mathrm{~b}-3$ was achieved in $75 \%$ of patients. The thrombectomy procedure failed in 10 cases. The median delay from MRI to recanalization was $132 \mathrm{~min}$ and the median delay from symptoms onset to recanalization was $327 \mathrm{~min}$.

\section{Outcome and safety}

Outcomes and safety of the thrombectomy group are shown in table 2.

One patient was lost to follow-up. Favorable clinical outcome (mRS score 0-2) at 3 months was more often achieved in patients in the thrombectomy group $(30 \%, 18$ patients) than in

\begin{tabular}{|c|c|c|}
\hline Outcomes & $\begin{array}{l}\text { Thrombectomy } \\
(n=60)\end{array}$ & Control $(n=48)$ \\
\hline Successful recanalization, $\mathrm{n}(\%)$ & $45(75 \%)$ & NA \\
\hline \multicolumn{3}{|l|}{ Clinical outcome at 90 days, n (\%) } \\
\hline $\mathrm{mRS} \leq 2$ & $18(30 \%)$ & $1(2.1 \%)$ \\
\hline $\mathrm{sICH}$ & $3(5 \%)$ & $3(6.3 \%)$ \\
\hline Malignant Infarction & $5(8.3 \%)$ & $9(18.8 \%)$ \\
\hline Hemicraniectomy & $2(3.3 \%)$ & $11(22.9 \%)$ \\
\hline Mortality & $15(25 \%)$ & $23(47.9 \%)$ \\
\hline
\end{tabular}

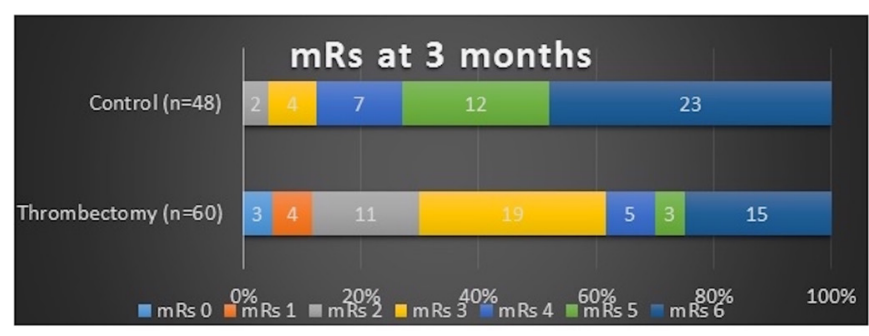

Figure 1 Modified Rankin Scale (mRS) score at 90 days in the thrombectomy and in the control groups. Scores on the $\mathrm{mRS}$ range from 0 to 6 , with 0 indicating no symptoms, 1 no clinically significant disability, 2 slight disability, 3 moderate disability, 4 moderately severe disability, 5 severe disability, and 6 death. There was a significant difference between the intervention group and the control group in the favorable distribution of scores defined by an $m R S \leq 2$ in analysis $(p<0.001)$.

the control group $(2.1 \%, \mathrm{p}<0.001)$. Scores on the mRS at 3 months in the thrombectomy group and in the control group are summarized in figure 1 .

Malignant stroke was observed in $18.8 \%$ of the patients in the control group versus $8.3 \%$ in the treatment group $(p=0.11)$. Craniectomy was performed in $22.9 \%$ of the control group versus $3.3 \%$ in the treatment group $(p=0.002)$. sICH was found in $6.3 \%$ of the patients in the control group versus $5 \%$ in the treatment group $(p=0.78)$. Mortality rates were $47.9 \%$ in the control group versus $25 \%$ in the treatment group $(p=0.01)$.

Only one of seven patients with atherothrombotic tandem occlusion in the thrombectomy group had a favorable outcome at 3 months, but four of these seven patients achieved TICI $2 b-3$ at the end of the procedure (two procedure failures). In the case of internal carotid dissection all patients had favorable recanalization, with three of four patients having a mRS score of $0-2$ at 3 months.

\section{Subgroup analysis}

\section{Age subgroup analysis}

Baseline characteristics and outcomes of the thrombectomy group according to age are shown in table 3.

The treatment group included 40 patients aged $\leq 70$ years $(67 \%)$ and 20 patients aged $>70$ years (33\%). Favorable clinical outcome (mRS $\leq 2)$ at 3 months was obtained in $37.5 \%$ of treated patients aged $\leq 70$ years compared with $10 \%$ for patients age $>70$, which is statistically significant $(p=0.02)$. This result was not related to median baseline NIHSS score (20 in the MT group vs 19.5 in control group; $\mathrm{p}=0.26)$, median baseline ASPECTS (5 in each group; $\mathrm{p}=0.64$ ), successful thrombectomy (75\% in each group; $\mathrm{p}=0.8)$ or mortality rate $(37.5 \%$ in the MT group vs $55 \%$ in the control group; $\mathrm{p}=0.53)$.

The median delay from onset of symptoms to the emergency room (131 min for those $\leq 70$ years vs $150 \mathrm{~min}$ for those $>70$ years; $p=0.91)$ or to MRI $(168 \mathrm{~min}$ for those $\leq 70$ years vs 154 min for those $>70$ years; $p=0.57$ ) was similar in the two groups. The median delay from MRI to recanalization was significantly shorter in patients $\leq 70$ years $(109 \mathrm{~min}$ vs $163 \mathrm{~min}$ for those $>70$ years; $p=0.02$ ) but the median time from onset of symptoms to recanalization was similar (336 min for those $\leq 70$ years vs $321 \mathrm{~min}$ for those $>70$ years; $\mathrm{p}=0.40$ ).

\section{ASPECT subgroup analysis}

We distinguished two ASPECT subgroups in our study population. One with a very low ASPECT score (0-3) and one with 
Table 3 Baseline characteristics and outcomes of the thrombectomy group $(n=60)$ according to age subgroup

\begin{tabular}{lll}
\hline & $\leq 70$ years $(\mathrm{n}=40)$ & $>70$ years $(\mathrm{n}=20)$ \\
\hline Median age (IQR) & $58(22-70)$ & $75(71-86)$ \\
\hline Median NIHSS score (IQR) & $20(9-27)$ & $19.5(10-27)$ \\
\hline $\begin{array}{l}\text { Median ASPECTS (IQR) } \\
\text { Median delay, min (IQR) }\end{array}$ & $5(2-5)$ & $5(3-5)$ \\
\hline $\begin{array}{l}\text { Onset of symptoms to admission } \\
\text { Onset of symptoms to MRI }\end{array}$ & $131(10-380)$ & $150(41-331)$ \\
\hline $\begin{array}{l}\text { Onset of symptoms to } \\
\text { recanalization }\end{array}$ & $168(20-392)$ & $154(64-346)$ \\
\hline $\begin{array}{l}\text { MRI to recanalization } \\
\text { Successful recanalization, } \mathrm{n}(\%)\end{array}$ & $336(153-488)$ & $321(227-504)$ \\
\hline Clinical outcome at 90 days, $\mathrm{n}(\%)$ & $109(73-253)$ & $163(52-382)$ \\
\hline $\begin{array}{l}\text { mRS } \leq 2 \\
\text { Mortality }\end{array}$ & $15(37.5 \%)$ & $15(75 \%)$ \\
\hline ASPECTS, Abs) & $15(37.5 \%)$ & $11(55 \%)$ \\
\hline
\end{tabular}

ASPECTS, Alberta Stroke Programme Early CT Score; IQR, interquartile range; mRS, modified Rankin Scale; NIHSS National Institute of Health Stroke Scale; sICH, symptomatic intracranial hemorrhage.

a low ASPECT score (4-5). In the thrombectomy group, most patients belonged to the subgroup with a low ASPECT score $(54 / 60 ; 90 \%)$. This subgroup of patients showed a $31.5 \%$ good clinical outcome close to that of the the overall group. In the control group 21 patients (44\%) had a low ASPECT score and a $4.7 \%$ good clinical outcome, with no statistical difference in the median ASPECT score ( 5 vs 4, respectively). Patients with a very low ASPECT score (0-3) and radiological-clinical mismatch are quite rare. Indeed, only six patients (10\%) underwent thrombectomy and only one of those patients had a good clinical outcome over the 6-year inclusion period compared with $0 \%$ in the control group, which included 27 patients (56\%) with a very low ASPECT score.

In the thrombectomy group, median delays from onset of symptoms to admission or MRI and from MRI to recanalization were similar in patients with a very low ASPECTS and in those with a low ASPECTS. However, the delay from onset of symptoms to recanalization was significantly lower in the very low ASPECTS group than in the low ASPECTS group $(\mathrm{p}=0.04)$.

\section{DISCUSSION}

Our study provides the following findings: (1) reperfusion therapy in patients with acute stroke in the anterior circulation and ASPECTS $\leq 5$ on DWI seems to be efficient and safe, contributing to a favorable clinical outcome at 90 days and reducing malignant infarction, hemicraniectomy and death. (2) Among these patients, those younger than 70 years are associated with a better clinical outcome.

In the recently published meta-analysis on behalf of the HERMES collaborators, ${ }^{9}$ Goyal and al pooled data from recent randomized control trials (RCTs), including MR CLEAN, SWIFT PRIME, REVASCAT, EXTEND IA, and ESCAPE. ${ }^{4-8}$ Their results showed a statistically significant benefit of thrombectomy in combination with IVT over IVT alone, with an increase in absolute benefit of $19.5 \%$ for a better clinical outcome and a positive odds ratio of 1.72 .

In RCTs ${ }^{4-8}$ patients had small infarction volumes with a median ASPECTS varying from 7 to 9 . Only 9.4\% of the HERMES $^{9}$ population had an ASPECTS between 0 and 5 . In this subgroup, analysis shows a tendency in favor of thrombectomy
$(\mathrm{OR}=1.24)$, but this was not statistically significant $(95 \% \mathrm{CI}$ 0.62 to 2.49 ). HERMES analysis ${ }^{9}$ suggests that lower baseline ASPECTS is strongly associated with lower rates of favorable outcomes. So, because most trials excluded patients with a low ASPECTS $\leq 5$, the effect of endovascular thrombectomy in this category of patients could not be established by RCTs.

Our study analyzed patients with large ischemic score at baseline on MRI/DWI with ASPECTS $\leq 5$. In the thrombectomy group, patients had severe baseline clinical and imaging characteristics with a median ASPECTS of 5, a median pretreatment NIHSS score of 20, and 22\% had a tandem lesion. Comparing our results with patients treated by mechanical thrombectomy in RCTs $^{4-8}$ and with the overall results of the HERMES meta-analysis, ${ }^{9}$ we noted the following findings. The favorable outcome of $30 \%$ in our thrombectomy group is slightly lower $(32.6-71 \%$ in RCTs; $46 \%$ for HERMES) and the mortality rate of $25 \%$ is moderately higher $(9-18.4 \%$ in RCTs; $15.3 \%$ for HERMES). In contrast our results are comparable to previous studies for successful recanalization rate $(75 \%$ vs $58.7-100 \%$ in RCTs; $71 \%$ for HERMES) and sICH (5\% vs $0-7.7 \%$ in RCTs; $4.4 \%$ for HERMES). In our study we selected patients with low ASPECTS so the baseline median ASPECTS was lower than in the RCTs (5 vs 7-9) and the baseline median NIHSS score was higher (20 vs $16-17$ in RCTs).

We must point out that in the RCTs, necrotic core volumes might have been underestimated owing to the chosen imaging modality. Indeed, MR CLEAN ${ }^{4}$ selected patients using a plain CT scan to rule out intracranial hemorrhage and to confirm large vessel occlusion. Our center uses MRI to screen patients with stroke. We are fully aware that the MRI DWI is more sensitive in the detection of ischemic core than CT scan ASPECTS, so it would be misleading to compare CT ASPECTS with ADC ASPECTS.

\section{Control group}

In our study, we compare a group of patients treated by MT with a control group consisting of patients recruited within the same period with an ASPECTS $\leq 5$ on MRI/DWI, who did not met our selection criteria for revascularization therapy. The outcome of these patients not treated was worse than that of patients treated by MT as shown by mRS score, level of malignant stroke, craniectomy, and death. No significant differences were found in the baseline characteristics of patients between the two groups for age, NIHSS score, site of arterial occlusion, and delay from onset of symptoms to emergency room presentation. Only ASPECTS was lower in the control group. Because ASPECT scores between these two groups are unadjusted, the two groups are not strictly comparable. Nevertheless, these results remain interesting since they give us a perspective on the natural history and outcome for patients with large strokes in the anterior circulation not treated by revascularization therapy. Moreover, the unfavorable outcome in untreated patients (2.1\% of patients with mRS $0-2$ at 3 months and $47.9 \%$ mortality) leads us to propose revascularization therapy in patients with extensive infarction core, especially whose with ASPECTS 4-5 (representing 90\% of our treated patients).

\section{Age subgroup}

Our results suggest that patients aged $\leq 70$ years in the thrombectomy group achieved a more favorable clinical outcome than patients aged $>70$ years, regardless of the characteristics of patients, especially the recanalization rate, which was similar in both age subgroups. 
These findings are corroborated by the results published in 2009 by Qureshi et al ${ }^{11}$ from a pooled analysis of four prospective studies showing that older patients are at higher risk of a poor outcome regardless of the recanalization rate. A different hypothesis had been suggested to explain this poor outcome. The impact of comorbidities on the outcome of patients with stroke was evaluated by Fischer et $a l^{12}$ who concluded that atrial fibrillation, coronary artery disease, and diabetes have a statistically significant impact on outcome. Lastly, in the HERMES meta-analysis, ${ }^{9}$ patients older than 80 years, benefit from thrombectomy with a favorable OR of 3.68 (95\% CI 1.95 to 6.92). But most of these patients had a small infarct volume, and therefore it is difficult to extend the results of RCTs to older patients with a large infarction core.

In view of these data, a decision for endovascular treatment in patients aged $>70$ years with ASPECTS $\leq 5$ should take into account a patient's other characteristics, such as comorbidities, cognitive status, and baseline autonomy.

\section{Study limitations}

Our study had several limitations. First, our data were from a single center with a retrospective analysis of our databank and the number of patients was quite small. Second, our center uses MRI to screen patients with stroke. So it seems misleading to compare ASPECTS on DWI and CT used in RCTs because a CT scan is known to be less sensitive in the detection of ischemic score. Third, ASPECT scores between the control group and the thrombectomy group are unadjusted.

\section{CONCLUSION}

Our preliminary results suggest that endovascular therapy can safely and efficiently be applied in patients with ischemic stroke with ASPECTS $\leq 5$ on admission (based on DWI and radiological-clinical mismatch). These patients should not automatically be recused from endovascular therapy, especially if they are aged $<70$ years. Older patients should be considered for thrombectomy individually and their comorbidities and pre-stroke status taken into account. Nevertheless, multicenter randomized trials are required to confirm these results and to determine the optimal multimodal imaging criteria for patient selection, especially for patient aged $\leq 70$ years.
Contributors Conception of the work: AB, VC. Acquisition of data: IM, EA, DM, TS, $\mathrm{XA}, \mathrm{OFE}, \mathrm{GG}, \mathrm{CD}, \mathrm{CR}, \mathrm{MM}, \mathrm{CA}$. Analysis and interpretation of data and drafting the work: IM, EA Edited the manuscript for intellectual content: VC, AB, MM. Revising manuscript critically for important intellectual content, final approval of the version to be published, and agreement to be accountable for all aspects of the work in ensuring that questions related to the accuracy or integrity of any part of the work are appropriately investigated and resolved: All authors.

Funding This research received no specific grant from any funding agency in the public, commercial or not-for-profit sectors.

Competing interests None declared.

Ethics approval Institutional review board.

Provenance and peer review Not commissioned; externally peer reviewed.

(c) Article author(s) (or their employer(s) unless otherwise stated in the text of the article) 2018. All rights reserved. No commercial use is permitted unless otherwise expressly granted.

\section{REFERENCES}

1 Vogt G, Laage R, Shuaib A, et al. Initial lesion volume is an independent predictor of clinical stroke outcome at day 90: an analysis of the Virtual International Stroke Trials Archive (VISTA) database. Stroke 2012;43:1266-72.

2 Jovin TG, Yonas H, Gebel JM, et al. The cortical ischemic core and not the consistently present penumbra is a determinant of clinical outcome in acute middle cerebral artery occlusion. Stroke 2003;34:2426-33.

3 Nezu T, Koga M, Kimura K, et al. Pretreatment ASPECTS on DWI predicts 3-month outcome following rt-PA: SAMURAI rt-PA Registry. Neurology 2010;75:555-61.

4 Berkhemer OA, Fransen PS, Beumer D, et al. A randomized trial of intraarterial treatment for acute ischemic stroke. N Engl J Med 2015;372:11-20.

5 Goyal M, Demchuk AM, Menon BK, et al. Randomized assessment of rapid endovascular treatment of ischemic stroke. N Engl J Med 2015;372:1019-30.

6 Jovin TG, Chamorro A, Cobo E, et al. Thrombectomy within 8 hours after symptom onset in ischemic stroke. N Engl J Med 2015;372:2296-306.

7 Saver JL, Goyal M, Bonafe A, et al. Stent-retriever thrombectomy after intravenous t-PA vs. t-PA alone in stroke. N Engl J Med 2015;372:2285-95.

8 Campbell BC, Mitchell PJ, Kleinig TJ, et al. Endovascular therapy for ischemic stroke with perfusion-imaging selection. N Engl J Med 2015;372:1009-18.

9 Goyal M, Menon BK, van Zwam WH, et al. Endovascular thrombectomy after large-vessel ischaemic stroke: a meta-analysis of individual patient data from five randomised trials. Lancet 2016;387:1723-31.

10 Hacke W, Kaste M, Bluhmki E, et al. Thrombolysis with alteplase 3 to 4.5 hours after acute ischemic stroke. N Eng/ J Med 2008;359:1317-29.

11 Qureshi Al, Suri MF, Georgiadis AL, et al. Intra-arterial recanalization techniques for patients 80 years or older with acute ischemic stroke: pooled analysis from 4 prospective studies. AJNR Am J Neuroradiol 2009;30:1184-9.

12 Fischer $U$, Arnold M, Nedeltchev K, et al. Impact of comorbidity on ischemic stroke outcome. Acta Neurol Scand 2006;113:108-13. 